\title{
Internet of Things-Aware Process Modeling: Integrating IoT Devices as Business Process Resources
}

\author{
Sonja Meyer ${ }^{1,2}$, Andreas Ruppen ${ }^{2}$, and Carsten Magerkurth ${ }^{1}$ \\ ${ }^{1}$ SAP Research, 9000 St. Gallen, Switzerland \\ ${ }^{2}$ University of Fribourg, 1700 Fribourg, Switzerland \\ sonj.meyer@sap.com, andreas.ruppen@unifr.ch, \\ carsten.magerkurthasap.com
}

\begin{abstract}
The Internet of Things (IoT) has grown in recent years to a huge branch of research: RFID, sensors and actuators as typical IoT devices are increasingly used as resources integrated into new value added applications of the Future Internet and are intelligently combined using standardised software services. While most of the current work on IoT integration focuses on areas of the actual technical implementation, little attention has been given to the integration of the IoT paradigm and its devices coming with native software components as resources in business processes of traditional enterprise resource planning systems. In this paper, we identify and integrate IoT resources as a novel automatic resource type on the business process layer beyond the classical human resource task-centric view of the business process model in order to face expanding resource planning challenges of future enterprise environments.
\end{abstract}

Keywords: Internet of Things, BPMN, Business Process Modeling, Resources, Sensors.

\section{Introduction and Motivation}

We believe that future advancements in web technologies will lead to a further integration of real-world entities into Internet applications. That means that smart devices will actively participate in the Future Internet. In 1999, the term "Internet of Things" was first used by Kevin Ashton concerning RFID tags in supply chains. In recent years, the IoT has become a buzz word. It comes with innumerable devices as potential resources being able to flexibly perform even parts of traditional business processes. Actual development activities target to define an architecture reference model to identify the main building blocks of the IoT. Following the work of [1], this reference model distinguishes between the blocks entity, device, native service and service. In this IoT world, connected devices ("temperature sensor") can interact with physical entities ("flowers") by its on device software resources ("sensing software component on sensor device") that can be accessed through standardized services ("Web Service Interface"). In order to bring this potential into enterprise applications, we aim at integrating the IoT with the monolithic architecture of ERP systems. 
But how does this IoT with its main building blocks fit in the conventional, traditional business process environment, which focuses on human resources and services hitherto known in the form of activities as steps in a predefined process flow?

Typical enterprise solutions such as ERP systems could benefit from the integration with the IoT, if business process-related devices such as RFID, sensors and actuators could directly take over responsibility as process resources for individual process tasks. From the process perspective, IoT devices stand out that they can interact with their physical environment in similar ways as human users. Furthermore, they acquire the ability to communicate exposing non-standard software components through services, which are accessible from the outside by web-like standards. We assume that the direct integration of intelligent devices on the one hand leads to a modification of existing business processes, and on the other hand entirely new business processes will arise.

Today's ERP systems provide, according to an applied procedure model e.g. [2], extensive mechanisms for the design, resolution, execution and monitoring of business processes. One important initial step of this predefined BPM lifecycle is the actual acquisition of the company's business process that shall be covered and auto-mated by the ERP system. The current industry standard BPMN 2.0 allows, besides the creation of a graphical process model, the generation of a machine-readable model. This model serves as an input for further cycle steps such as the usage of a BPMN 2.0 compliant resolution and execution engine [3].

This paper contributes the solution to the problem that so far the role of IoT devices as a resource type of a business process is not directly represented in standard process models. This means: The IoT devices and their non-standard software components do not exist as process resources from the perspective of ERP systems! This implicates that without the proposed solution, IoT devices cannot be considered in the automatic resolution phase as potential execution responsible parties and no duties can be assigned to them. With falling technology prices and the advancing IoT research branch it is expected that soon business processes will emerge that require considering IoT devices in the process flow as resources both for documentation and automation purposes. It is therefore important to establish early a process metamodel that covers this new requirement. With this research contribution, future ERP systems shall obtain a process model basis which they can apply to efficiently plan and use devices of the IoT according to their availability in addition to traditional resources such as human staff for the operational enterprise processes.

This work investigates how the component "IoT device" and its native services can be expressed as a resource in an IoT-aware process model. Thus, we present five main contributions:

- First, we analyze and identify those IoT domain concepts which take over a resource role from business process perspective.

- Second, we depict the IoT device and its underlying native software components as a combination of swim lane and process activity-centric resource model. 
- Third, we integrate a general semantic model that captures the requirements to later BPM phases of IoT devices and its native software components as parameters.

- Fourth, we extend the graphical stencils and both the CMOF representation and the XML schema of the process metamodel of the notation BPMN 2.0. On the semantic side we integrate the work from [4] and [5].

- Last, we test the proposed resource model practically: Therefore, we implement a prototype of the model and extend the web-based editor of [6]. The result is discussed in terms of an example process.

\section{Background Information}

Current ERP systems focus on modeling and executing planned business processes in an enterprise environment with a constant number of process resources such as human performers in a company. In contrast to that, the IoT comes with thousands of devices as potential resources in a web like structure being able to flexibly perform parts of business processes and quickly react with adapted processes to a permanent changing environment. In order to bring that potential into enterprise applications, we aim at integrating the IoT with an existing Business Process Management (BPM) environment. Business Process Management (BPM) is defined as "a systematic approach to capture, execute, measure, document, monitor and control automated and non-automated processes to reach certain goals" [7]. One central part and the basis of the lifecycle before any process automation, is the creation of a Business Process Model. Today's ERP systems provide a loosely-coupled approach based on the Service Oriented-Architecture (SOA) paradigm and assuming that a business process is composed out of exchangeable process tasks. A process task corresponds to a unit of work implemented by a service [8]. In the IoT domain it is distinguished between rather traditional web services [9] and a certain class of services called IoT services making use of native software components on IoT devices (e.g. sensors and actuators). In order to bring these new types of IoT devices as a potential resource in the envisioned BPM environment, we aim to provide an IoT-aware Business Process Model, as a basis to express all process relevant IoT information in. To build our work on a scientific foundation, we have investigated existing states of Business Process Notations. We focus on conservative modeling standards for complementing existing business processes by straightforward IoT integration in order to maximize a potential industrial application and foster a widespread adoption similar to the adoption of core web technologies on the Internet. In [10], BPMN 2.0 was evaluated as the most IoT-aware state of the art process modeling approach, besides the most commonly used process notations EPC, UML activity diagram and WS-BPEL. Such, BPMN will serve as basis for the mapping work presented in this paper. The process model comprises a graphical and a machine readable representation. Following [11] who distinguish between the professional and technical process model we aim to provide the technical model as the more detailed and executable mode. Therefore, based on [12] we consider the process model as a set of IoT and non-IoT process tasks having resolution and execution restrictions between these tasks. The central 
outcome of the process design is the IoT-aware business process model serving as a clearly defined interface between the process design and deployment phase. It has to be decided which process constructs are used to represent and handle typical and all possible IoT situations in the process model. We therefore define IoT device and native service constructs and the decision for them as modeling conventions of BPMN 2.0.

\section{Business Process View on IoT Domain Model}

\subsection{Overall Component Perspective}

This section defines the main abstractions and concepts underlying the IoT domain which are important from a business process view and describes the relationships between these components. To simplify, we first reduce and subsume, similar to [4], the IoT Domain Model of the IoT-Architecture ${ }^{1}$ to its core components that are central to business process modeling. Building on [13], [14] we define the following major components:

- IoT service: Software components with well-defined and standardized interfaces, which enable access to other, heterogeneous components with native interfaces and expose their functionality as a unit of work to a business process.

- Physical entity ${ }^{2}$ : Identifiable separable part of the physical environment, which is of central interest for a user or an application, such as a business process.

- IoT device: Technological artifacts that can interconnect the physical world with the digital world by the provision of projection capabilities such as monitoring, sensing or actuating. It comes with communication capabilities to other IT systems and either belongs to a physical unit, or is directly integrated into it.

- Native service ${ }^{3}$ : Software components with native interfaces hosted on IoT devices that allow users or applications such as a business process, to gather information about entities or perform actions on entities in its physical environment.

The various relations between the different components are shown in Fig. 1. A physical entity of the physical world can be associated with an IoT service. This connection is the result of the relations between the other model components. Thus, the actual source of information of the IoT services is the native software component that is accessed. An IoT service consequently exposes a standard interface to the functionality of one or more native software components, which thereby become usable in a business process. Correspondingly, the relation between the native service and a physical entity results from obtaining the relations between the other components of the model. The IoT device hosts native software components that contain executable

\footnotetext{
${ }^{1}$ http: / / www. iot-a.eu/public/

${ }^{2}$ The term Thing/Object is also vicarious used in the Internet of Things.

${ }^{3}$ In the IoT-A terminology ${ }^{1}$ the component "native service" is called "resource", but from a process perspective this term already refers to the process participant being responsible for assigned execution units, named process activities.
} 
code. Using its non-standard interfaces these native services may on the one hand provide information to the IoT services and on the other hand they may obtain information from the IoT services that allows for performing certain physical actions. Between an IoT device and the digital representation of a physical entity the artificial relationship "attached to" is created, so that the physical entity becomes part of the digital world through using an IoT device. Properties of the entity can in consequence be measured or changed by the IoT device.

Continuing to integrate the view of the information model of [13] with respect to the defined specification models, there is the possibility to assign each major component to a knowledge base that defines describing information in a structured form, and expresses their logical relations to one another. Ontologies are usually applied to describe an existing knowledge base. As part of the process model, however, it is suitable to use the same ontologies to formulate requirements for individual elements of the process model as they are used for the process resolution phase- the actual allocation of process elements. For instance, for this replaceable description model the OWL definitions of [5] or the USDL definition of [15] might be applied.

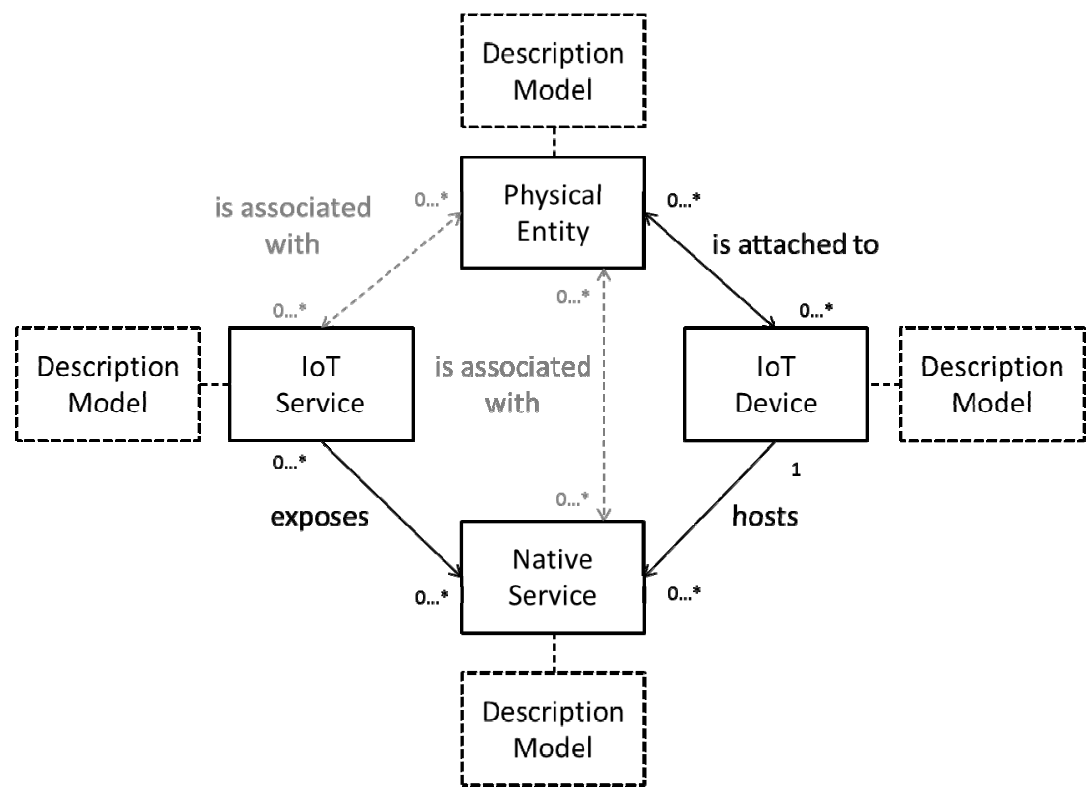

Fig. 1. Business process relevant IoT domain components (own presentation based on [13] and [5])

\subsection{Resource Perspective}

This section includes mapping the identified, modeling relevant IoT components of the last section to the typical available components that are provided by business process modeling notations. Here we focus on the identification of the components as process resources. For this general part we don't focus explicitly on the BPMN 2.0 notation, even though some components are not available in other modeling notations. 
For the resource definition from the process perspective we reflect the following four main components:

- A physical entity is, following [16] and [17], a necessary part of the process model, as it is indispensable for the correct resolution and execution of the process. We consider the example of changing the price of the product, in our example an orchid in a supermarket depending on its temperature, as presented in [10]. If the information "physical entity orchid" is not available in the process model, it is unclear which product shall be given a price reduction in case of increasing temperature values in the supermarket, and the process using a process model could neither be fully mapped on nor be executed correctly. Nevertheless, in contrast to typical process-participants, the physical entity has no process execution responsibility. Therefore, it is not a process resource and only indirectly participates in the process flow. Process participants who have no responsibility to execute any activity or whose activities are simply unknown, and who don't have any process flow, are considered as a kind of "black box" or passive process participant.

- The IoT service definition can be combined with the definition of an activity (respective action, function) as it exists in many process notations ${ }^{4}$. An activity in the process model means a unit of work that is performed in the process. If this activity is performed by a resource, and the internal subpart is not describable by the process model, it is considered to be atomic or named "task" in [18]. A fully automated software component with a standardized interface as the IoT service could though be represented as a task. To address a separate functionality during the resolution of the process model that is specific to the IoT service, it is advised in [17] to establish a separate subclass. Summing up, a service is not a process resource, but a unit of work to which a resource can take over execution responsibility.

- An IoT device as a technical artifact can offer computing resources to a process and acts similarly to a human user as a linking artifact between the process and the real world. Analogously to a human process resource, the IoT device as a technical process resource is responsible for the execution of activities and thus it adopts a direct role as a separate process performer in the model. From the perspective of the process model, an IoT device can be understood as a resource so that it contains in itself sub-resources - namely software components with native interfaces.

- Following the IoT domain model, a software component with a native interface cannot become part of a process without the help of an additional software component coming with well-defined and standardized interfaces that can expose it. It is not yet covered by existing process models, neither as a sub-task of the task nor as a sub-resource of the device performer. It is hence an indirectly used software component that is accessed through the well-defined service interface that already presents an atomic unit in the model and does not foresee any underlying unit. From a process model perspective, we understand a native service as a process resource hosted on an IoT device that performs the actual deployment responsibility and according to Fig. 1 has the relation ,,is exposed" to the process task.

\footnotetext{
${ }^{4}$ For instance, BPMN, WS-BPEL, EPC, UML Activity Diagram and Petrinets.
} 
As summarized by Fig. 2, two out of four main components of the IoT Domain Model fall in the perspective of business process resources: the IoT device and the native service. For both components description parameters can be specified applying the description of model of [5], which is relevant for the process resolution and execution. The BPMN 2.0 notation basically supports two ways to represent resources in the process model: one, on activity-level and one, on cross-activity-level. In the following we will apply both of these options to the IoT domain model.

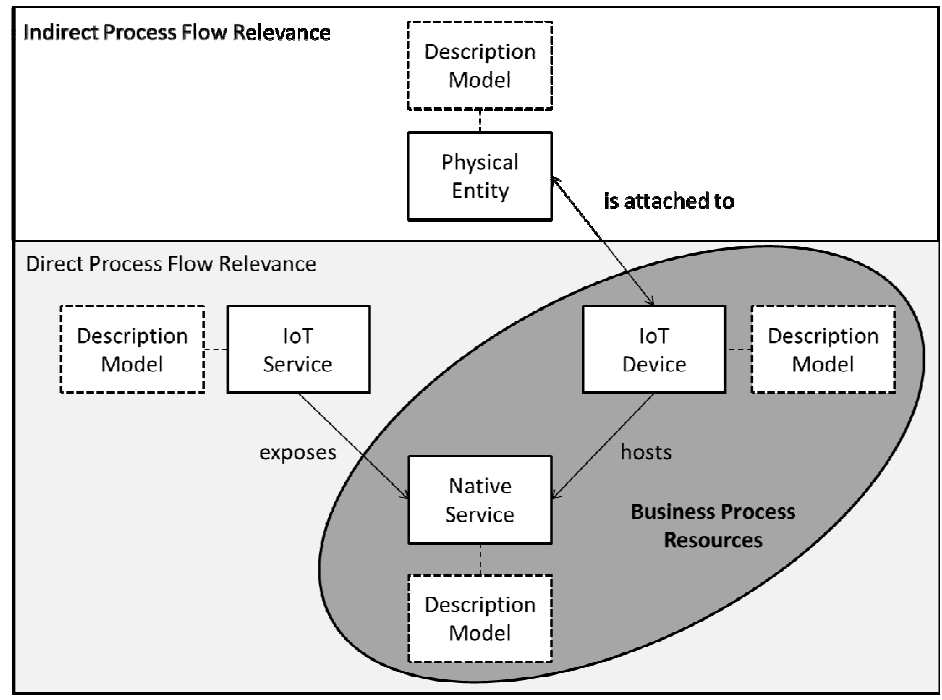

Fig. 2. Classification of IoT components in business process view

\section{IoT Devices and Natives Services as Process Resources in BPMN}

In this section, we propose a specification of the IoT device and its native software components as an extension to the BPMN 2.0 notation. When integrating the identified business process resources of the last section to BPMN, we face two main challenges. First, an IoT device and its native service are usually not considered and there-fore they are not mentioned in the specification. The reason for this is that one central ERP system is often assumed to automate all enterprise business processes without including millions of distributed and connected devices, all coming with the capability to directly overtake execution responsibility. Second, we have to deal with two types of process resources on different levels at the same time, the IoT device and its hosted native services being exposed by an IoT service. Usually the BPMN 2.0 standard considers different roles of resources, but not two completely different types 
on different abstraction levels concurrently in the same process model. The BPMN standard comes with different representations: a graphic model and a machinereadable model consisting of a CMOF specification and an XSD specification. We have split this section in three parts: An overview, a graphical model and a machine-readable model section.

\subsection{General Overview}

An IoT device is a technical process performer. In contrast to the physical entity it is a process-internal artifact similar to a human user that directly participates in the process flow. In the background, it takes over the execution responsibility of the native software components behind the standardized IoT services. In this manner, an IoT device is not managed by a Process Execution Engine and has no direct interface to the Execution Engine. Functional and non-functional requirements of the device can be defined, which can be understood and taken into account during the resolution of the process model, the actual service allocation. The challenge is that we are dealing at the same time with a second process resource, namely the native software component on the device. Also the native service denotes a process resource. While for human resources, from the process point of view, it is not distinguished between a person and its abilities in relation to the process execution, for real-world resources this distinction is made.

\subsection{Graphical Model}

A process lane is an optional subdivision of a pool, the container for a process, in order to associate process activities with particular actors, departments or roles, but it is not restricted to these categories. [19] Traditional flowcharting is used to indicate the performer role or owner of activities. So like shown in Section 4.2 a lane is not a purely graphical element, but semantic element in its own. In order to explicitly distinguish an IoT Device from conventional process performers and to bind the IoT Device specific description model as specifiable attributes to its graphical element, we use a characteristic icon in the lane, similar to the proposed handling of mobile phones of [20]. Fig. 3 shows a process pool called "IoT Process" containing the regular lane "Lane" and the IoT device "IoT Device". The second task of the graphical model is an IoT specific "Sensing Task" exposing the actual native sensing service of the IoT device. Consequently, the native service is not directly visible as part of the graphical process model, but included in the IoT task representation. To the right an extended version of the associated description model is shown, which can be used to further specify the element "IoT Device" of "Native Service". The description model is not directly part of the graphical model, but the realization of the graphical attributes in a tree-like structure depends on the respective editor tool implementation. 

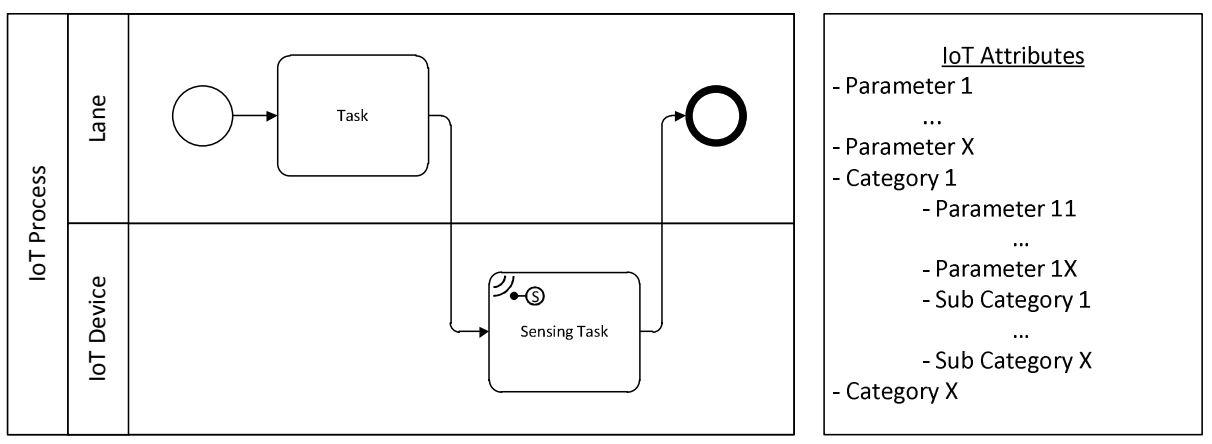

Fig. 3. IoT Device being responsible for a sensing task in a process pool / description model

\subsection{Machine-Readable Model}

This section defines how an IoT device can be represented as a process performer by expanding the lane element of the BPMN 2.0 machine-readable model and introducing a new subclass. Additionally, we expand the resource role in order to map the process resource's native service to an activity level by staying as standard compliant as possible. Finally, we present a concept that can refer to external parameter description schemas and is used by the two new classes.

The UML class diagram in Fig. 4 illustrates the CMOF representation with the IoT specific extensions. Accordingly, a process in [18] is defined as a subclass of a flow element container. The process is therefore a container for one or more sets of lanes, each of them serving again as a container for one or more lanes. A Lane as a partition element may reference multiple FlowNodes such as Activities, Events, or Data Objects. Each of the Lanes of dedicated Lansets can contain a BaseElement, such as the resource role. BPMN 2.0 already supports the allocation of resources at activity level. Per activity an arbitrary number of resource roles may be defined. The class performer is a subclass of ResourceRole and thus inherits all the attributes and model associations. It defines the resource that performs an activity. For our purposes we define, as BPMN foresees, the specific NativeService Performer class in parallel to the existing HumanPerformer class.

Through the corresponding introduction of a new resource role as a subclass IoTDevice, as the standard requires, we face three problems which we discuss in the following:

- Defining a new resource element results in the deletion of any assigned resources to other elements in the lane such as to the activities. That means the standard does not support two simultaneous process resource definitions as needed by the IoT domain on lane level as well as on activity level.

- BPMN supports internally known process resources, but no external parameterbased resource definitions that are based on ontologies. In other words, BPMN can only reference parameters for a resource role, which is known in XML format to the BPM environment. Descriptions for IoT elements are not bound to an XML 
based BPMN standard, but can include diverse description schemas which should be directly referenceable as well.

- The same problem follows with the non-parameter-based, prior allocation of a specific process resource on lane level, e.g. a specific temperature sensor.

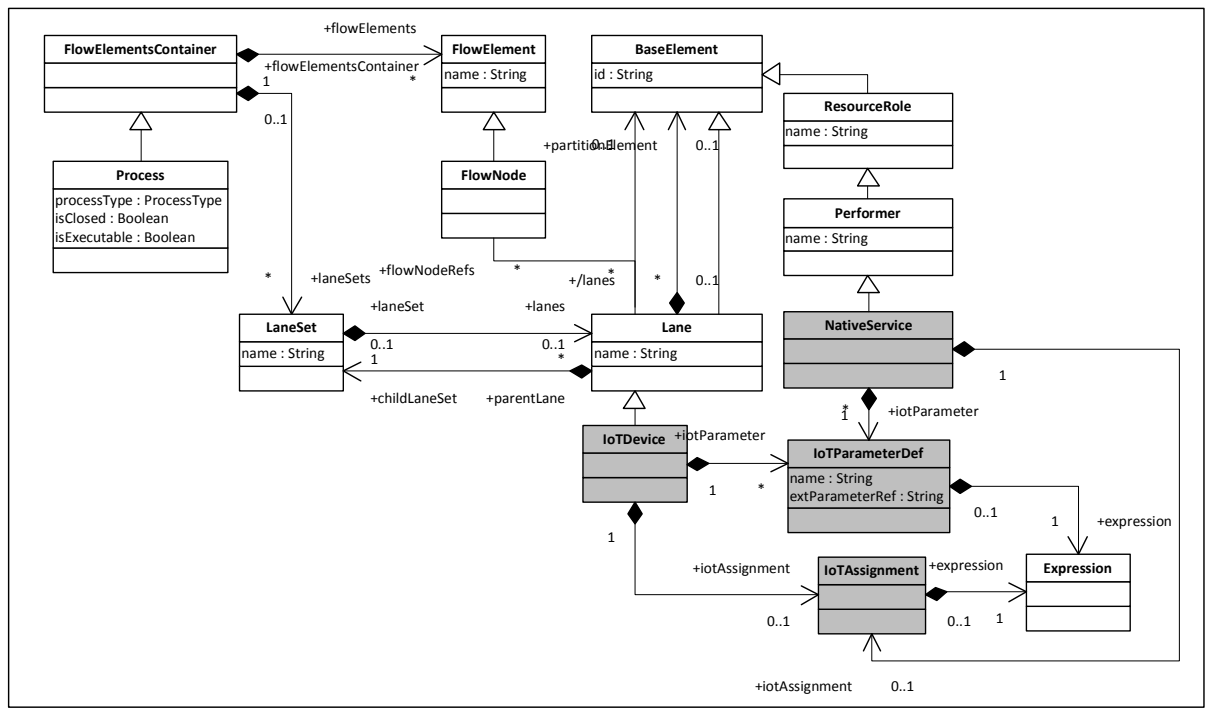

Fig. 4. IoT specific class extension of CMOF representation of BPMN 2.0 specification

To overcome these problems, we propose to introduce a subclass to Lane called IoTDevice and thus to bring the resource allocation of devices directly to the lane level, compatible to the graphical model. The subclass inherits the relationships and attributes of the class Lane and thus, those of the class BaseElement. If a device is then defined above its enclosed activities, it no longer excludes the definition of service requirements or requirements to the native service component and at the same time applying the conventional BPMN standard to them. The new element IoTDevice supports the definition of diverse parameters using the IoTParameterDef class. The parameters with its values pass over the generated XML document to the resolution phase of the process, so that at runtime an available device might be found that meets the requirements defined by the parameters. Which of and how the defined parameters are finally taken into account for the real allocation is decided by an infrastructure component as envisioned by [21]. The schema of the parameters is not stored as part of the BPMN model, but referenced with extParameterRef pointing to individual sheets of a corresponding ontology that is specialized to the schema description of an IoT device or native service 5 . The standard class Expression can capture the actual parameter value belonging to IoTParameterDef, which the process resource shall fulfill.

\footnotetext{
${ }^{5}$ E.g. http: / / ccsriottb3 .ee.surrey.ac.uk:8080/IotaDataFiles / models/ResourceModel.owl
} 
IoTAssignment defines the Expression that is used for the resource assignment, both for the IoT device as well as for the native service. This assignment can be either made already at the time of process modeling, if a dedicated available resource is known beforehand, or it can thus bind the result of the infrastructure resolution component to the process model. Consequently, a process resource can be defined as a potential performer including its desired properties either directly in the lane (IoT device) or as part of an activity (native service), but without being known previously to the BPM environment. The actual assignment of activities can be rolled out to an external infrastructure component.

\section{$5 \quad$ Modeling Resource Extensions in Process Example Using Extended Editor}

In the previous section we have discussed our approach of presenting the IoT domain components IoT device and native service as process resources in the business process model by extending the standard BPMN 2.0. To illustrate the benefit of the proposed approach, we discuss our realized implementation of extending a web-based editor tool in terms of a real-world business process example. Our modeling tool ${ }^{6}$ based on [6] integrates the discussed concepts and thus enables Business Process Modeling Experts to create IoT devices and native services in business processes as modeling elements, what was not supported so far without our proposed extensions.

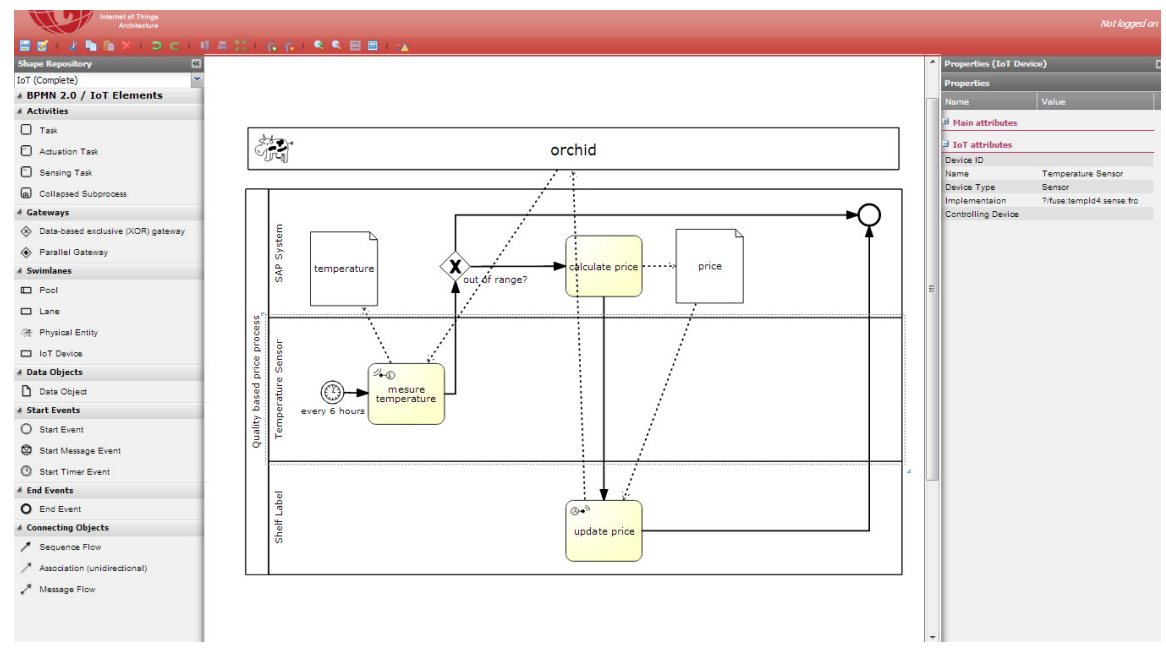

Fig. 5. Dynamic pricing process with the two IoT Devices temperature sensor and ESL

We envision a dynamic pricing process in the retail domain showing how the IoT device temperature sensor monitors the perishable good orchid in a store. The measurement of the temperature sensor is used to estimate the quality of the good and the

\footnotetext{
${ }^{6}$ Available at http: / / www . iot $4 \mathrm{bpm}$. de
} 
price, which indicated on an electronic shelf label (ESL), is automatically reduced if the temperature rises. Fig. 5 shows the corresponding graphical model in the IoTaware Business Process Modeling tool. The process includes two IoT devices: a temperature sensor offering its on IoT device sensing components through a standardized IoT service "measure temperature" and an ESL offering its on device actuating components through the service "update price". The IoT devices and its native services can now be defined declaratively at modeling time as an own lane subtype. For letting the business process be resolved and deployed automatically by the envisioned BPM environment, in which the actual available IoT devices change constantly, the process modeler can influence the dynamic resolving process by further specifying individual device or native service parameters as requirements. For avoiding that any improper device associated to the orchid of the IoT is assigned as temperature sensor to this business process through the resolution component, the modeler can add multiple requirements to the new IoT elements. Due to certain business arrangements of his company, he is obliged to deploy exclusively devices of the manufacturer "Future Store". Additionally, the native service of the temperature sensor shall provide a temperature accuracy of $0.1^{\circ} \mathrm{C}$ since the product orchid already reacts to smallest temperature variations. Both properties can now be defined in the process model by the established standard extensions. The annotations are not only added graphically, but they are rather written in an extended BPMN 2.0 XML document, which is the machine-readable output of the modeling activity and serves as input for the deployment phase. The XML putput follows from the demonstrated CMOF extensions of Chapter 4, which were applied to the BPMN 2.0 XML schema specification in the tool. Below an excerpt of the XML code is shown containing the parameter assignment to the IoT device.

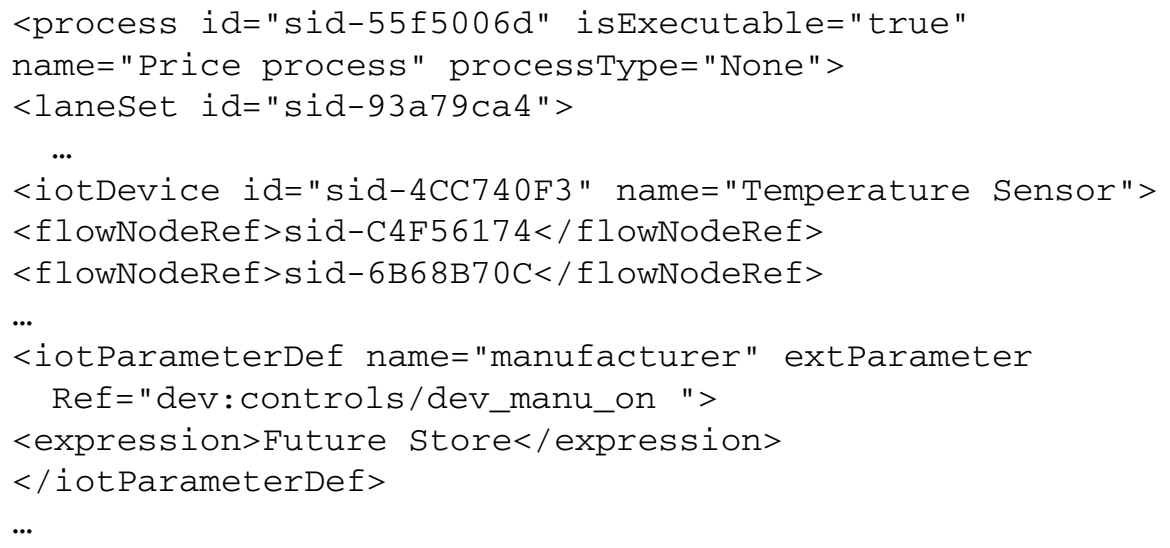

We have simplified shown in this section, how IoT devices and its native services can be modeled and expressed in a dynamic pricing process using a web-based Business Process Modeling Tool. This implements our extensions to the notation of BPMN as one initial part of a future BPM-based ERP-System, that shall include the vision of the IoT in order to enable thousands of smart IoT devices to take part as a new resource type in a business process. 


\section{Discussion}

The integration of the two process resources of the IoT Domain model presented in the last sections shows one way how to represent sensors and actuators directly in a business process model without contradicting to existing standards. Thereby the abstraction of the native software component is assumed by a service with a standardized interface that implements an atomic process task in a process model. In addition to the Internet of Things, there is a related research initiative, the "Web of Things" [22], posed by a complete integration of IoT devices to the Web, which central ideas were not yet covered by our work. It assumes that physical entities provide knowledge through IoT devices to the web with the help of REST interfaces. In this context, it is often not a service-oriented architecture (SOA) that is envisioned and on which most of today's BPM solutions are based on, but a resource-oriented architecture is assumed, where an information unit is seen as a resource of evolutionary cycle. Presumably, efforts as [23], [22] could be partially considered in the information model of business processes. Nevertheless, such an approach is mostly useful when SOA remains the central idea of the underlying system architecture, by which business processes are realized. In our view, the near future of today's BPM-based IT systems will face the challenge of dealing with this information-centric resources perspective.

\section{$7 \quad$ Related Work}

This section compares our proposed solution with the related work on the resource view of sensor and actuator-based business processes of the Future Internet. We point out differences and similarities between the approaches. Existing approaches are generally still in their infancies and concentrate either solely on the graphical model of business processes [24],[20], the integration of data artifacts [24], [25] or the automated code generation for sensor networks [26], but not on the mapping of the central process resources from the IoT domain to a standard business process model. The work of [24] presents a graphical syntax to BPMN, in order to model and publish RESTful business processes. The focus lies on the restful interaction as a special message flow type between a dedicated process activity and the persistent data source. Our solution focuses on a pre-defined process flow without technical details of interaction that is resolved through an infrastructure component, where services are dynamically bound to the process model, be it based on SOAP or REST principles. We see the communication principles not as constructs of the modeling notation, but as part of the service support of the execution tool implementation. Similar to [24], [25] employs an information-centric approach to model business processes. The "resources" of the REST Architectures are not process activity instances but information entities. This new information-centric approach does not consider process resources in the performer responsibility, but centers on data resources. Our approach focuses on the integration of existing service-based technologies as used in traditional BPM-based ERP system today. [26] discusses how synchronization primitives 
expressed in BPMN can be transformed to efficient event-based code running on resource-constrained sensor nodes. While Caracas concentrates on sensor nodes, our approach allows for categorizing any IoT devices among further process performer roles so that both the process model and the resolution component can identify and handle IoT devices and their native software components appropriately. Thus, an appropriate resolution technique can be used and the typical IoT situation that several devices are obtainable for the potential execution of a specific process can be resolved. [20] introduces possible extensions to BPMN in order to express the mobility of devices in a process as part of the graphical model. It is suggested to extract the mobile participant out of the organization's pool and to use a separate pool or lane for the mobile device. Our solution combines the approach of [20] with the style rules of [19] and considers multiple devices in one common business process pool as a lane.

\section{Conclusion and Outlook}

The lack of modeling concepts to represent sensors and actuators of the IoT and their native software components in a business process model is a significant obstacle to successfully include real-world resources in business processes of traditional ERP systems supporting a BPM lifecycle. In this paper, we have shown how to improve this situation considerably with a new extension to standard process modeling approaches that introduces IoT devices as a process resource type to the business process meta-level. In order to reach that goal, we initially introduced the IoT device and its underlying native software components to the process metamodel and came up with a general semantic model for capturing resource allocation requirement to process elements. For demonstrating the purpose of the new resource type we accordingly extended the three layers of the process metamodel coming with the standard BPMN 2.0 notation. Furthermore, we mapped the ontologies coming with the IoT description model of [5] to the introduced sematic model of BPMN 2.0. Finally, we tested the proposed resource model practically by implementing a prototype of the model and thus extending the web-based editor of [6].

Our future work will include a broader application of the IoT Reference Architecture presented in [13]. Further research will deal with the stepwise implementation of the identified IoT- specifies of Section 3 in the business process modeling domain and beyond.

Acknowledgments. The authors would like to thankfully acknowledge the support for this work provided by the European Commission within the FP7 project IoT-A, contract number: 257521. The authors would also like to thank Surpana De and Payam Barnaghi for providing their work on IoT description models as input.

\section{References}

1. Haller, S.: The things in the internet of things. In: Poster at the (IoT 2010), Tokyo, Japan (November 2010)

2. Scheer, A., Cameron, I.: Architecture of integrated information systems: foundations of enterprise modelling. Springer, Heidelberg (1992) 
3. Activiti BPM Platform. Activiti. (2012)

4. De, S., Barnaghi, P., Bauer, M., Meissner, S.: Service modelling for the Internet of Things. In: Federated Conference on IEEE Computer Science and Information Systems, FedCSIS (2011)

5. De, S., Elsaleh, T., Barnaghi, P., Meissner, S.: An Internet of Things Platform for RealWorld and Digital Objects. Scalable Computing: Practice and Experience 13(1) (2012)

6. Signavio Core Components. Signavio GmbH (2012)

7. ABPMP: Business Process Management Common Body of Knowledge - BPM CBOK. Leitfaden für das Prozessmanagement. Verlag Dr. Götz Schmidt (2009)

8. Leymann, F., Roller, D., Schmidt, M.: Web services and business process management. IBM Systems Journal 41(2), 198-211 (2002)

9. Booth, D., Haas, H., McCabe, F., Newcomer, E., Champion, M., Ferris, C., Orchard, D.: Web services architecture (2004)

10. Meyer, S., Sperner, K., Magerkurth, C., Pasquier, J.: Towards modeling real-world aware business processes. In: Proceedings of the Second International Workshop on Web of Things, p. 8. ACM (2011)

11. Freund, J., Rücker, B., Henninger, T.: Praxishandbuch BPMN. Hanser (2010)

12. Weske, M.: Business process management: concepts, languages, architectures. SpringerVerlag New York Inc. (2007)

13. Walewski, J.: Initial Architectural Reference Model for IoT. EC FP7 IoT-A Deliverable $1.4(2012)$

14. Thoma, M., Meyer, S., Sperner, K., Meissner, S., Braun, T.: On IoT-services: Survey, Classification and Enterprise Integration. In: IEEE International Conference on Internet of Things 2012, Besançon, France (2012)

15. Martin, G.: Resource Description Specification. EC FP7 IoT-A Deliverable 2.1 (2012)

16. Sperner, K., Meyer, S., Magerkurth, C.: Introducing entity-based concepts to business process modeling. In: Dijkman, R., Hofstetter, J., Koehler, J. (eds.) BPMN 2011. Lecture Notes in Business Information Processing, vol. 95, pp. 166-171. Springer, Heidelberg (2011)

17. Meyer, S.: Concepts for Modeling IoT-Aware Processes. EC FP7 IoT-A Deliverable 2.2 (2012)

18. Business Process Model And Notation (BPMN). OMG Specification. Object Management Group (2011)

19. Silver, B.: BPMN method and style. Cody-Cassidy Press (2009)

20. Kozel, T.: BPMN mobilisation. In: Proceedings of the European Conference of Systems: World Scientific and Engineering Academy and Society, WSEAS (2010)

21. De, S.: Concepts and Solutions for Entity-based Discovery of IoT Resources and Managing their Dynamic Associations. EC FP7 IoT-A Deliverable 4.3 (2012)

22. Guinard, D., Trifa, V., Wilde, E.: A resource oriented architecture for the web of things. In: Proc. of IoT (2010)

23. Bizer, C., Heath, T., Berners-Lee, T.: Linked data-the story so far. International Journal on Semantic Web and Information Systems (IJSWIS) 5(3), 1-22 (2009)

24. Pautasso, C.: BPMN for REST. In: Business Process Model and Notation, pp. 74-87 (2011)

25. Kumaran, S., Liu, R., Dhoolia, P., Heath, T., Nandi, P., Pinel, F.: A restful architecture for service-oriented business process execution. In: IEEE International Conference on eBusiness Engineering. IEEE (2008)

26. Caracas, A., Bernauer, A.: Compiling business process models for sensor networks. In: 2011 International Conference on Distributed Computing in Sensor Systems and Workshops (DCOSS). IEEE (2011) 